\title{
Haptic Interaction Techniques for Exploring Chart Data
}

\author{
Sabrina A. Panëels ${ }^{1}$, Jonathan C. Roberts ${ }^{2}$, and Peter J. Rodgers ${ }^{1}$ \\ 1 Computing Laboratory, University of Kent, \\ CT2 7NF Canterbury, UK \\ \{sap28,p.j.rodgers\}@kent.ac.uk \\ 2 School of Computer Science, Bangor University, \\ Dean Street, Bangor Gwynedd LL57 1UT, UK \\ \{j.c.roberts\}@bangor.ac.uk
}

\begin{abstract}
Haptic data visualization is a growing research area. It conveys information using the sense of touch which can help visually impaired people or be useful when other modalities are not available. However, as haptic devices and virtual worlds exhibit many challenges, the haptic interactions developed are often simple and limited to navigation tasks, preferring other modalities to relay detailed information. One of the principal challenges of navigation with haptic devices alone, particularly single point-based force-feedback devices, is that users do not know where to explore and thus obtaining an overview is difficult. Thus, this paper presents two types of interaction technique that aim to help the user get an overview of data: 1) a haptic scatter plot, which has not been investigated to any great degree, provided by a force model and 2) a new implementation for a haptic line chart technique provided using a guided tour model.
\end{abstract}

Key words: Haptic Visualization, Haptic Interaction Techniques, Haptic Scatter Plots, Haptic Line Charts

\section{Introduction}

The use of non-visual forms of data communication, including haptics, is rapidly increasing as low cost devices become more available. Haptic technology is not only useful to increase accessibility but also provides an alternative modality to convey information (for instance when the other senses are overloaded, to provide a sensory duplication of the information or to provide different information from other modalities). Consequently, one growing application area is haptic data visualization: the use of tactile or force-feedback devices to represent and realize information and data [1]. Haptic data visualization aims to provide an alternative 'perception' or understanding of the underlying data through effective data mappings and user interactions using the sense of touch. Through the interactions with such a representation, the user can gain quantitative or qualitative understanding of some underlying data. 
Therefore, interaction techniques hold a key role in the interaction with and experience of the virtual world. There are several challenges of navigating haptic 3D worlds: haptics provides a lower perceptual bandwidth than vision, especially when many haptic devices are single point tools, and thus the haptic environment lacks the detail of spatial awareness that is implicit in vision. Consequently, finding new suitable metaphors and effective interaction techniques is essential, especially in the haptic visualization area, where they help the user gain an understanding of the data.

The use of haptic interactions in visualization is not widespread, as the field is young, and often involves simple interactions where haptics is limited to navigation and the auditory modality is used to convey information. This is particularly the case for charts applications. Indeed, charts are above all a visual medium as users can easily get an overview, spot trends and locate maximum and minimum values or other specific values. As the haptic modality has a much lower perception bandwidth than vision, perceiving a chart haptically is a more difficult task. For example, as most devices are single point-based, it is difficult to get an overview. Additionally, as one can lose its spatial awareness easily in a virtual $3 \mathrm{D}$ world, it becomes hard to make comparisons or realize values. Therefore, the challenge is to find effective representations and efficient interaction techniques that will enable understanding the underlying data.

This paper presents two new haptic interaction techniques for charts to help the user get an overview of the data: the visualization of a haptic scatter plot and a haptic line chart. Our scatter plot technique models the plot by assigning a repulsive force to each point. The user explores the plot and greater force is felt for larger concentrations of data points. For the line chart method, we implement a guidance tour, based on a museum tour metaphor first suggested by Roberts et al. [2]. Both techniques have been implemented using the forcefeedback PHANTOM device.

Little research has been carried out for the haptic visualization of scatter plots. Haase and Kaczmarek [3] designed and tested the display of scatter plots both on a fingertip- and an abdomen-based eletrotactile display and conducted an experiment evaluating them. Scatter plots were created from sampling bivariate normal distributions with 25 data points and no axes were represented. Crossan et al. [4] on the other hand presented a visualization method based on haptic textures produced using granular synthesis and using the force-feedback PHANTOM device so that the user explores the textured surface that represents the plot. In contrast, our method for haptic visualization of scatter plots assigns a force depending on the distance to each point, meaning the user has a greater opportunity to explore the plot in $3 \mathrm{D}$, and can detect the forces from multiple directions.

As line charts are a common representation form, their non-visual representation has previously been investigated for the exploration and extraction of features. Initially, the line was represented by a cylinder or an embossed ridge, however, users found it difficult to follow the line without slipping off the edges [5], [6]. Fritz and Barner [5] used attraction forces to keep the user on the line. 
However, Yu et al. [6] proposed that engraved modelling should be used instead (also used by Van Scoy et al. [7]). Representing different lines is still an open challenge. Yu et al. [6] used different surface friction to distinguish the lines, but this misled the users at line intersections. In addition, using gridlines to convey coordinate values, as with the visual counterpart, was found to be confusing. Hence Yu et al. [8] adopted a multimodal approach, leaving haptics for navigation and dedicating the auditory modality to providing quantitative values through synthesized speech and overview through sonification. Roberts et al. [2] investigated the haptic overview and suggested (but did not implement) several different methodologies of exploration: unguided exploration (for instance a 'Friction \& Zoom View' where the areas above and below the lines were assigned different textures), the constrained exploration view (i.e. the 'Exploded Data View' provides the user with different and simplified views of the data) and the guided tour. In this paper we give implementation of our version of the guidance tour.

The remainder of this paper is organized as follows. In section 2 we explain in detail our scatter plot technique. In section 3 we show an implementation of the guidance tour metaphors for the line chart in a data flow prototyping tool. Finally, in section 4 we give our conclusions and discuss further work.

\section{Scatter Plots}

A scatter plot is a visualization technique used to reveal the correlation between variables. By displaying data as a collection of points, the scatter plot shows the relationship between the variables through the size and location of the point cloud, the direction of the relationship, and whether outliers exist. Analyzing a scatter plot typically consists of two tasks: identifying the general trend (direction, size and position) and spotting interesting features such as outliers. This process corresponds to the visualization mantra of Ben Shneiderman namely 'Overview, zoom and filter, details on demand'. Getting an overview is therefore the first step to understanding the data. Hence we designed an overview haptic interaction technique for scatter plots that conveys the general trend of the plot.

The interaction technique was developed using a haptic interaction prototyping tool (HITPROTO) based on the haptic scene-graph H3D API [9]. The tool uses visual configurable blocks to represent the flow of data. There are two main blocks categories: the flow blocks (Wait For, Everytime, Switch) which are either event-based (e.g. "Wait For the device's button to be pressed") or testing conditions ("If the key pressed is equal to 1") and the actions blocks (adding/removing effects, creation/modification of haptic and guidance effects). Setting the parameters of these blocks and linking them together enables to produce an executable scenario. $\mathrm{X}^{3} \mathrm{D}^{3}$ scenes can also be 'loaded' to use a scene

\footnotetext{
${ }^{3} \mathrm{X} 3 \mathrm{D}[10]$ is an ISO open standard scene-graph design and the successor to the VRML standard. It is used and extended within H3D to specify the 3D scene-graph of the world and particularly the geometric and basic haptic properties.
} 
representation or act on it (for example by highlighting shapes or removing them from the scene).

Figure 1 shows the data flow diagram of the scenario for the haptic visualization of a scatter plot. The data used was the Iris dataset by R.A. Fisher [11]. Three species of Iris flower (Setosa, Versicolor and Virginica) have been studied and particularly for the following parameters: sepal width, sepal length, petal width and petal length. ${ }^{4}$ Both $2 \mathrm{D}$ and $3 \mathrm{D}$ charts were generated to highlight the correlation of the flowers sepal length and petal length/width (see Figure 2 ). The scenario's concept is simple, each dataset is associated with a key on the keyboard (' 1 ', '2', '3') and pressing that key 'adds' the haptic effect to the corresponding dataset (see Figure 1). The user can then feel the datasets successively as well as the whole dataset. We believe that in doing so the user will get a general idea about the location of the different datasets relative to each other as well as their respective size as it provides simplified and different views of the data [2].

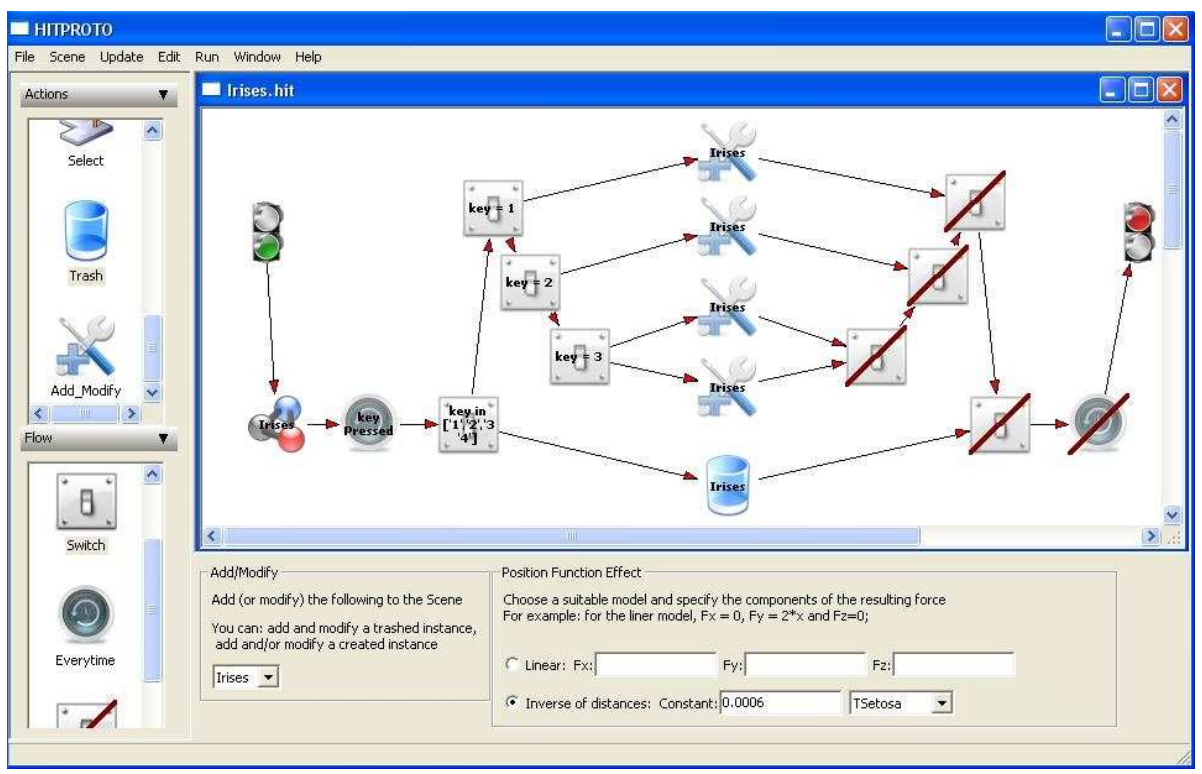

Fig. 1. Diagram showing the data flow of the scatter plot visualization of Irises. The haptic effect is first created, then when a key is pressed, the haptic effect is either set for a particular grouping node (for all of the Irises or each of them) or removed depending which key was pressed.

The force model used is part of the PositionFunctionEffect block in the HITPROTO tool where the user can either specify the 3D components of the force

${ }^{4}$ The Iris dataset was obtained from the XmdvTool website, in the data sets category[12]. 

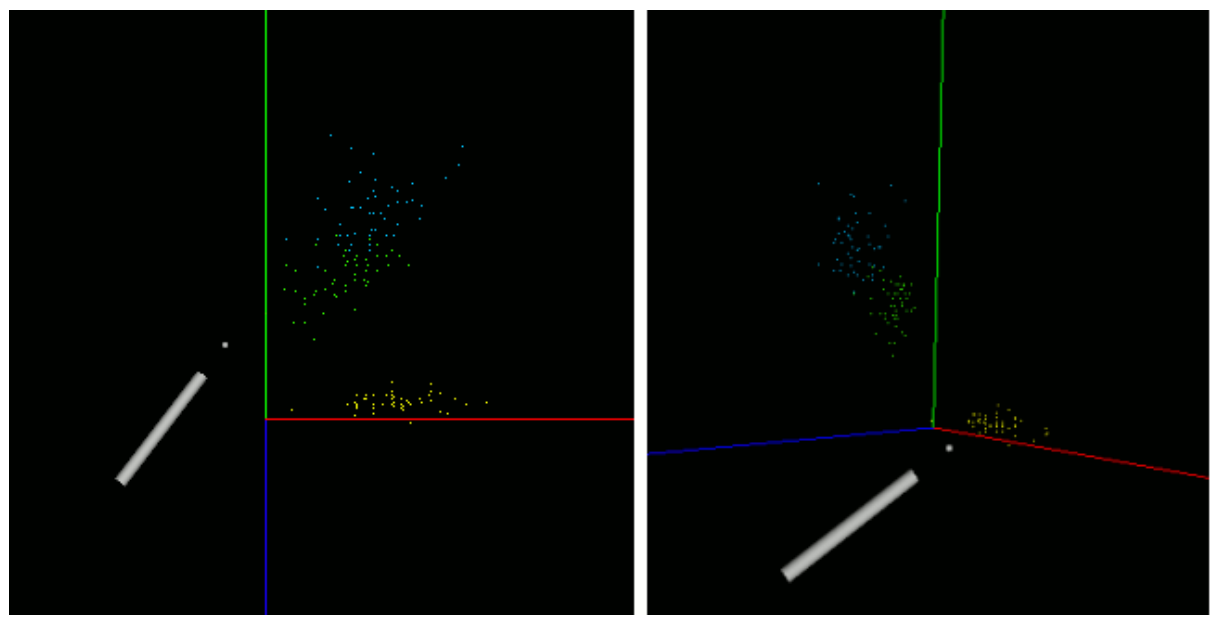

Fig. 2. Screenshot showing the 3D visual display of the Iris datasets used for the haptic visualization as well as a rotated view.

or use a predefined force model, which can be applied to grouping nodes in the scene graph, according to the device's position. For the scatter plot scenario, the predefined model was used (see Figure 3 and 4), which computes the resultant repulsive force to be applied as the sum of the inverse of the distances from the haptic device to each point from the point cloud in the chosen grouping node along with the sum of the unit vectors between the device and these points (see Equation 1 and Figure 3 for an example).

$$
\boldsymbol{F}=-k \times \sum_{i=1}^{n} \frac{1}{d_{i}} \times \boldsymbol{u}_{\boldsymbol{i}}
$$

with $d_{i}$, the distance from point ${ }_{i}$ to the device and $\boldsymbol{u}_{\boldsymbol{i}}$, the unit vector of the vector from the device to point $i$.

The initial idea to convey the scatter plot's general trend was a function that depends on the number of points within a given radius. However, simply using the number of points as a factor led to jerky forces thus highlighting the need for a continuous force that would give a smoother feeling. After experimenting and discarding different models (including one trying to use the device's velocity vector), we decided to use all the points of the dataset, and not only the ones present in the device's tip vicinity, along with the continuous model based on the inverse of the distances from each point to the device. The idea was that the closer the device would get to a highly point-concentrated area, the greater the force factor would be, while being further away from the points reduces the force (see Figure 4). This model can convey relatively well the direction of the point cloud and relative position to the other datasets (when felt successively as 


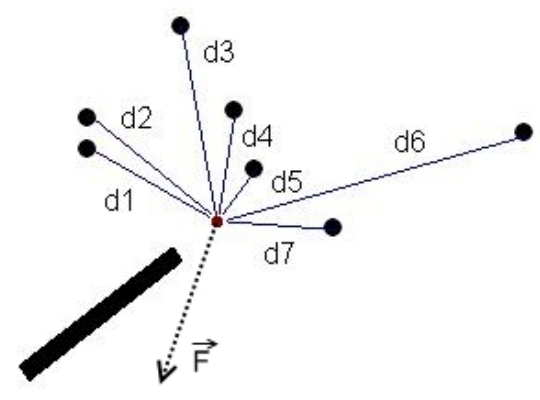

Fig. 3. Diagram describing the force model used in the scatter plot visualization scenario. The resultant force is equal to: $\boldsymbol{F}=-k \times \sum_{i=1}^{7} \frac{1}{d_{i}} \times \boldsymbol{u}_{\boldsymbol{i}}$ with k a constant and $d_{i}$ being the distances to the points from the device and $\boldsymbol{u}_{\boldsymbol{i}}$ the unit vectors from the device to the points.

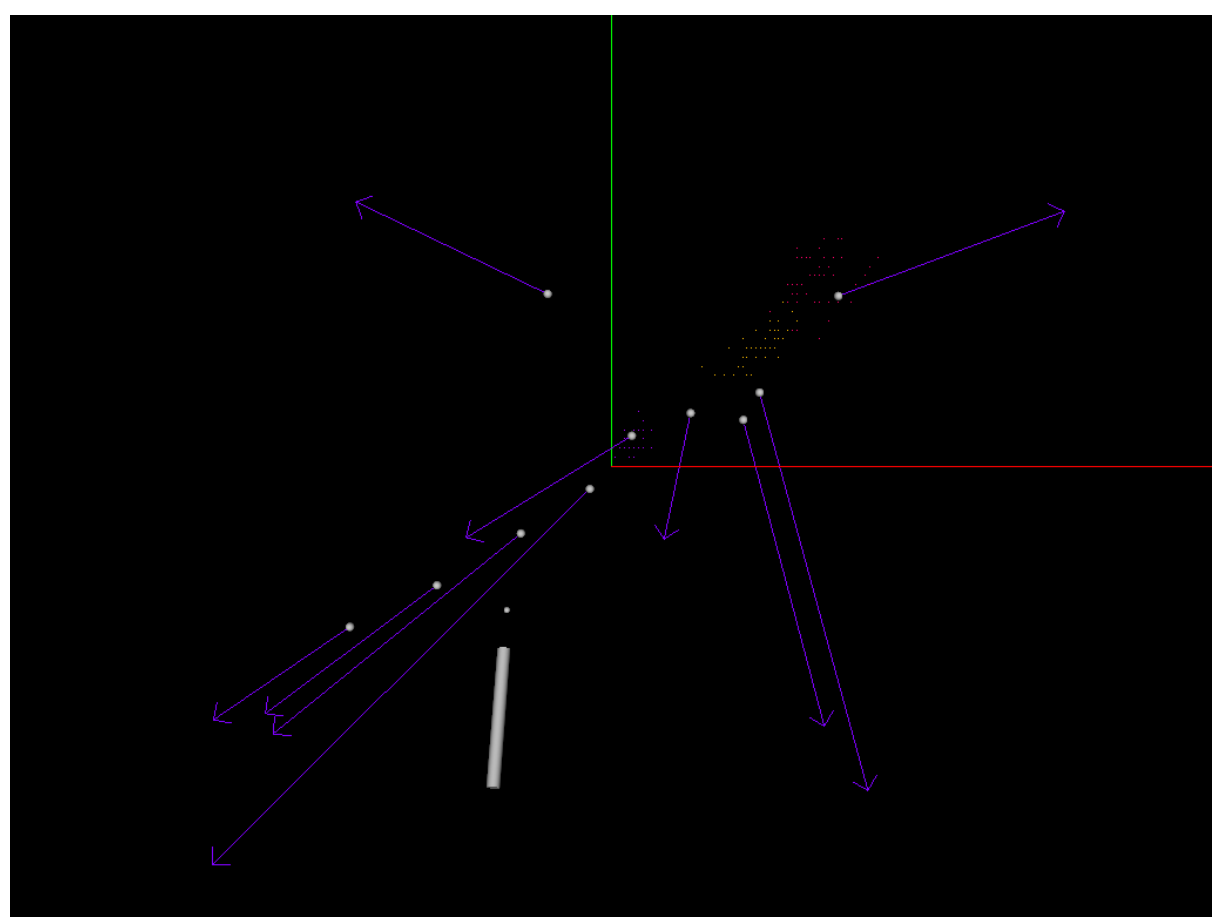

Fig. 4. To illustrate the forces on the device, this figure gives a $2 \mathrm{D}$ representation of the force model using a 2D Iris dataset. The larger filled circles show the device positions and the vectors show the force applied on the device. The magnitude of the force was scaled down for the figure. 
in the scenario presented above and in Figure 1). However, it is often difficult to get an accurate picture of the point cloud density.

The axes are not represented haptically and thus cannot be used directly with the scatter plot representation as the user can get lost easily in 3D space. Nonetheless, the axes could be included using a guidance interaction technique, as detailed in the following section, by taking the user to the centre of the axes and then to the first point(s) of the point cloud to help the user locate the point cloud within the world coordinate system.

\section{Line charts}

Line charts are one of the most common representations for statistical data. However, many challenges still remain for their exploration with non-visual techniques. Researchers resort to solving these using the auditory modality (as explained in section 1), however this modality may not be appropriate or available, and so a pure haptic technique would be preferable as an alternative. The challenges are similar to that for the scatter plot: getting an overview of the chart and locating and comparing specific features (such as minimum and maximum values, intersections). Getting an overview haptically is difficult, especially when using point-based devices.

We believe that guidance coupled with free exploration can contribute to building a better mental image of the chart. To that effect, three different guidance tours have been developed (initially presented as a poster at the HAID'07 workshop [13]) using the HITPROTO tool. In this paper, we describe an alternative implementation of the museum tour metaphor, where the user is taken along a predefined path and stops at chosen points of interest. The user can then roam around to explore the surroundings for a given amount of time before the tour resumes. The tour relies on a spring force to keep the user attached to a moving 'anchor'.

Unlike previous work ([6], [8]), the representation chosen occupies the whole chart display (not only positive values) and in addition to the V-shaped line, some 'bumps' are added at the intersections of the V-shape line with the axes (see Figure 5). Figure 6 shows the data flow diagram of the museum tour scenario. The museum tour interaction is the combination of a standard guidance block and the behaviour at points of interests during the movement. The guidance block enables setting general parameters, such as the parameters for the attaching force, the path and speed or cycle time (see Figure 6 as these general parameters are repeated in the 'Add_Modify' block). The behaviour at the points of interest is tuned with the block 'Everytime', set on monitoring the movement positions, the block 'Switch' testing whether the user is passing through a point of interest and the blocks 'Guidance_Control' to specify the actions of pausing (with the roaming around) and resuming the guidance. Additionally in this case, the axes are removed during the guidance and added back whenever the guidance is interrupted (by pausing it or by triggering the free exploration mode). 


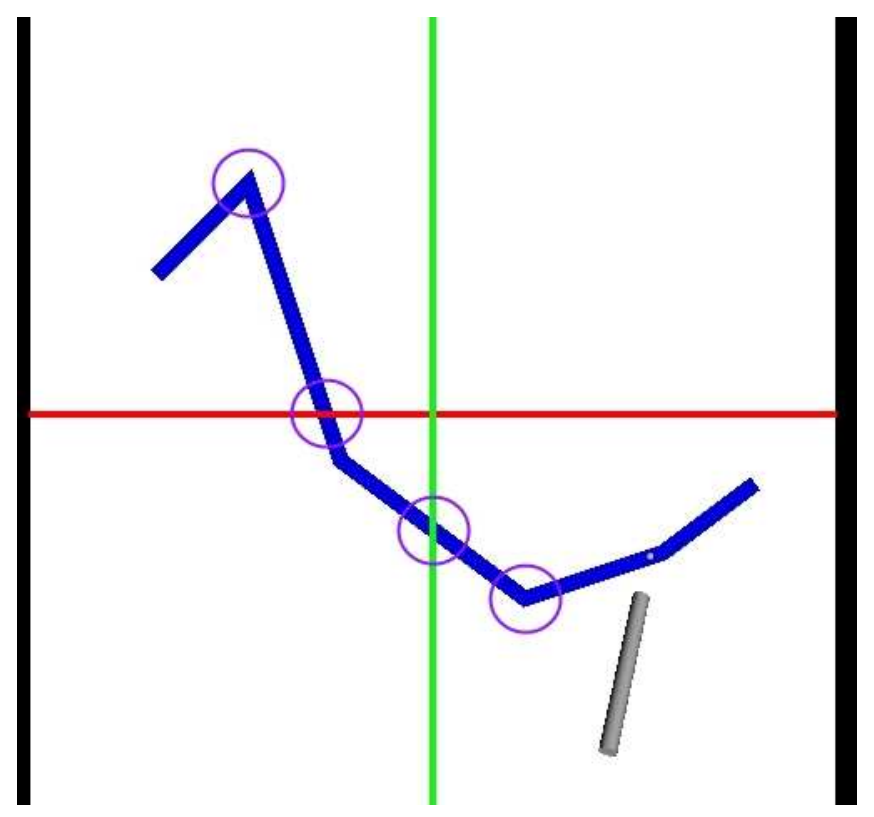

Fig. 5. The $3 \mathrm{D}$ visual display of the line chart, presenting a V-shaped line and embossed axes on a chart surrounded by walls. The device is led along the shown engraved line, but stops for a given time to allow for user exploration in the areas indicated by circles, which are max, min points and intersection with axes. 


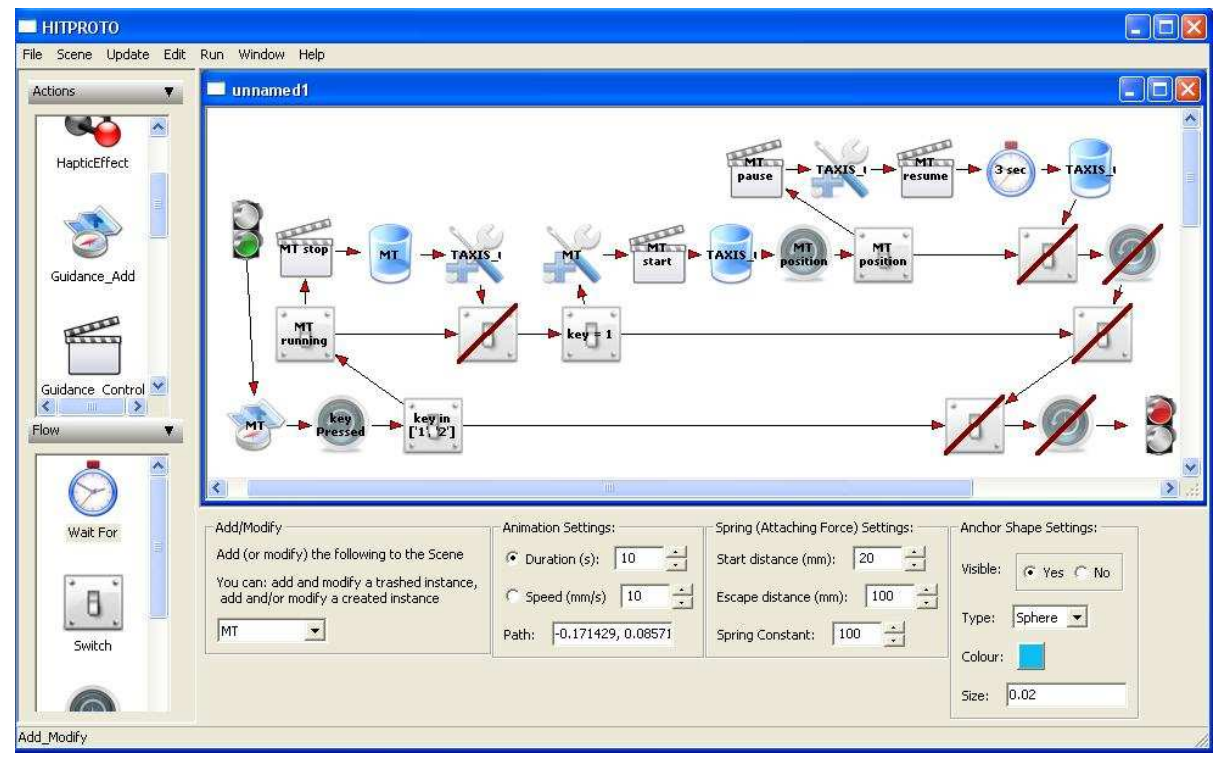

Fig. 6. Diagram showing the data flow of the museum tour scenario in the case of a line chart visualization. The tour stops at all the points of interests: maximum, minimum and intersection with the axes.

\section{Conclusion}

This paper has presented two types of interaction technique that aim to help the user get an overview of data. The first technique displays scatter plot data and uses a force model based on the sum of the inverse of the distances to each point from the current user location to feel the data. The interaction technique scenario described allows feeling the different datasets successively, thus providing the user with the relative positions of the datasets and their general direction. The second technique describes guidance tour interactions, in particular a museum tour metaphor, in the context of line chart data. The user is taken along the $\mathrm{V}$-shaped line and can explore the surroundings at points of interest, such as minima/maxima and intersections with the axes, where the tour pauses for a given time. Different variations of the guidance interactions (waterskier tour, bus tour, museum tour) can be used to provide different types of information (the whole line overview, the points of interests, the surroundings).

Future work might consist in perfecting the force model for the scatter plot interaction technique, as the ones presented lack accuracy for the point density and possibly finding and testing new models such as one using the inverses of the squared distances as commonly used in physics. Equivalent models could be found for tabular data. As for the guidance interaction techniques, more behaviour at the points of interest will be investigated in the case of line charts. Examples of such behaviour ideas include adding magnetic lines leading to the 
axes to provide an estimate of the coordinate values as well as possibly allowing the user to leave haptic landmarks to find the points easily during free exploration. The guidance techniques could also be extended to other types of charts, such as bar charts, where the path could take the user along each bar and back to the $\mathrm{x}$-axis or along the envelope of the bar chart. The two interaction techniques described should also be evaluated. More generally, other types of interaction techniques should be researched to provide chart overview and also to provide quantitative and qualitative values.

\section{References}

1. Roberts, J.C., Panëels, S.A.: Where are we with Haptic Visualization?. In: WorldHaptics, 2nd Joint EuroHaptics Conference and Symposium on Haptic Interfaces for Virtual Environment and Teleoperator Systems, pp. 316-323. IEEE Press, Washington DC (2007)

2. Roberts, J.C., Franklin, K., Cullinane, J.: Virtual Haptic Exploratory Visualization of Line Graphs and Charts. In: Bolas, M.T. (ed) the Engineering Reality of Virtual Reality. Electronic Imaging Conference, vol. 4660B, pp. 10-19, IS\&T/SPIE (2002)

3. Haase, S.J., Kaczmarek, K.A.: Electrotactile Perception of Scatterplots on the Fingertips and Abdomen. Med. Biol. Eng. Comput. 43, pp. 283-289 (2005)

4. Crossan, A., Williamson, J., Murray-Smith, R.: Haptic Granular Synthesis: Targeting, Visualisation and Texturing. In: 8th International Conference on Information Visualisation, pp. 527-532. IEEE Press, Washington DC (2004)

5. Fritz, J.P., Barner, K.E.: Design of a Haptic Graphing System. In: the Rehabilitation Engineering and Assistive Technology Society of North America Conference. RESNA (1996)

6. Yu, W., Ramloll, R., Brewster, S.A.: Haptic Graphs for Blind Computer Users. In: Brewster, S.A., Murray-Smith, R. (eds) Workshop on Haptic Human-Computer Interaction. LNCS, vol. 2058, pp. 102-107. Springer, Heidelberg (2000)

7. Van Scoy, F.L., Kawai, T., Darrah, M., Rash, C.: Haptic Display of Mathematical Functions for Teaching Mathematics to Students with Vision Disabilities: Design and Proof of Concept. In: Brewster, S.A., Murray-Smith, R. (eds) Workshop on Haptic Human-Computer Interaction. LNCS, vol. 2058, pp. 31-40. Springer, Heidelberg (2000)

8. Yu, W., Cheung, K., Brewster, S.A.: Automatic Online Haptic Graph Construction. In: Wall, S.A., Riedel, B., Crossan, A., McGee, M.R. (eds) EuroHaptics, pp. 128-133. (2002)

9. the H3D API, http://www.h3dapi.org/

10. the X3D Standard, http://www.web3d.org/

11. the Data and Story Library (DASL), http://lib.stat.cmu.edu/DASL/Stories/ Fisher'sIrises.html

12. XmdvTool Datasets, http://davis.wpi.edu/ xmdv/datasets/iris.html

13. Panëels, S., Roberts, J.C.: Haptic Guided Visualization of Line Graphs: Pilot Study. In: Workshop on Haptic and Audio Design, poster session. (2007) 